myotonic dystrophy. J Child Neurol April 1992; $\underline{7}: 161-167$.$) (Correspondence:$ Dr. P. Ian Andrews, Box 3533, Duke University Medical Center, Durham, NC 27710.)

COMMENT. These findings have relevance in genetic counseling and suggest that maternal age and birth order are important in the relative severity of childhood onset myotonic dystrophy. The authors note that a normal infant born after a neonatally affected child is unlikely to develop myotonic dystrophy.

An increased risk of spontaneous abortion, cesarian section and neonatal death for women with myotonic dystrophy is reported from the Chicoutimi Hospital, Quebec Canada (Prevost C, Mathieu J. Pregnancy outcome of women with myotonic dystrophy. Neurology April 1992; 42 Suppl $3: 226)$. The risk estimate that women with myotonic dystrophy will have a congenitally effected child is $3-9 \%$. Among children surviving the neonatal period, $7.3 \%$ had congenital myotonic dystrophy and $7.3 \%$ had childhood onset myotonic dystrophy.

\title{
DYSTROPHINOPATHY IN FEMALES
}

Dystrophin immunocytochemistry was used to study muscle biopsies from 505 girls and women with neuromuscular disease seen at the National Institute of Neuroscience, Tokyo, the University of Pittsburgh School of Medicine and the Columbia Presbyterian Medical Center, New York. The laboratory findings of $25(54 \%)$ of 46 isolated cases with muscle biopsies were consistent with the familial cases and all showed myopathic histopathology and abnormal elevations of serum CK. The clinical presentations of the isolated cases were consistent with familial cases and included proximal limb weakness before age $10(40 \%)$, myalgias or cramps $(24 \%)$ and grossly elevated CK levels (24\%). About $10 \%$ of the isolated cases of hyperCKemic myopathy were proven by dystrophin analysis to have a dystrophinopathy as the cause of their disease (manifesting carriers of Duchenne dystrophy). (Hoffman EP et al. Dystrophinopathy in isolated cases of myopathy in females. Neurology May $1992 ; \underline{42}: 967-975$.) (Reprints: Dr. E.P. Hoffman, University of Pittsburgh School of Medicine, Biomedical Science Tower, Pittsburgh, PA 15261; Dr. K. Arahata, National Institute of Neuroscience, 4-1-1 Ogawahigahi-cho, Kodaira, Tokyo 187, Japan; or Dr. E. Bonilla, Department of Neurology, ColumbiaPresbyterian Medical Center, New York, NY 10032.)

COMMENT. All isolated female dystrophinopathy patients had had other diagnoses prior to dystrophin testing, the most common being the limb girdle muscular dystrophy. The clinical criteria that implicate a dystrophinopathy in isolated nonfamilial cases in girls and women include a serum CK level in excess of 1,000 IU/l and a myopathic histopathology. The exclusion of dystrophinopathy by dystrophin analysis is necessary in confirming a diagnosis of isolated limb girdle muscular dystrophy.

A dystrophin test is also recommended in patients with idiopathic cardiomyopathies since cardiomyopathy was found as the only clinical manifestation in 2 Duchenne muscular dystrophy carriers reported from 
Rome, Italy (Mirabella M et al. Neurology April 1992; $\underline{42}$ Suppl $3: 281$ ). There was no muscle weakness and serum CK was only mildly increased.

\section{HEREDITARY MOTOR AND SENSORY NEUROPATHY MUTATION}

A duplication in chromosome 17 responsible for most cases of autosomal dominant HMSN 1 was present as a de-novo mutation in 9 out of 10 sporadic patients examined at the Department of Neurology, Academic Medical Center, Amsterdam, The Netherlands. (Hoogendijk JE et al. De-novo mutation in hereditary motor and sensory neuropathy type 1 . Lancet May 2, 1992; 339:1081-82.) (Correspondence: Dr. JE Hoogendijk, Department of Neurology, Academic Medical Center, Meibergdreef 9, 1105 AZ Amsterdam, The Netherlands.)

COMMENT. The previous assumption that isolated cases of HMSN I are most frequently autosomal recessive appears to be incorrect and testing for the duplication in chromosome 17 is essential to establish the mode of inheritance for genetic counseling of isolated patients.

This duplication of part of chromosome 17 was found in affected individuals from 7 of 8 families with HMSN I. Patients with HMSN type II do not show the duplication (Hallam PJ et al. Ann Neurol May 1992; 31:570-572). (Correspondence: Dr. Malcolm, Molecular Genetics Unit, Institute of Child Health, 30 Guilford St., London WC1N 1EH UK.)

HMSN type I also known as Charcot-Marie-Tooth disease type I is known to be genetically heterogeneous. At least 5 genetic loci have been identified including 3 dominant genes and $2 \mathrm{X}$-linked recessive genes. Ionasescu VV et al. from the Department of Pediatrics, University of Iowa Hospitals, Iowa City and the Massachusetts General Hospital present a clinical and genetic linkage study of 8 families with $\mathrm{X}$-linked dominant Charcot-Marie-Tooth neuropathy which supports a localization of the diseased gene between DXS14 and DXYS1 (Neurology April 1992; $\underline{42: 903-908) . ~}$

\section{SEIZURE DISORDERS}

\section{TEMPORAL LOBECTOMY FOR INTRACTABLE SEIZURES}

The results of temporal lobectomy in 16 children under the age of 12 years who had intractable seizures of temporal lobe origin are reported from the Miami Children's Hospital, Florida. All had seizures for at least 2 years despite multiple antiepileptic drugs. Structural lesions were demonstrated by neuroimaging in 11 patients. At follow-up from 1 to 4 years 11 children were seizure free, 3 were $90 \%$ improved, 1 was $50 \%$ improved and 1 was unchanged. Of 9 families contacted 7 reported substantial improvement in the child's psychosocial function. Neuropathological abnormalities identified in all children showed findings consistent with disordered neuronal migration in 7 , tuberous sclerosis in 1 , and mesial temporal sclerosis only in 2 children. (Duchowny $M$ et al. Temporal lobectomy in early childhood. Epilepsia 